\title{
Integrated Treatment of Aqueous Extract of Solanum nigrum-Potentiated Cisplatin- and Doxorubicin-Induced Cytotoxicity in Human Hepatocellular Carcinoma Cells
}

\author{
Chien-Kai Wang, ${ }^{1,2,3,4}$ Yi-Feng Lin, ${ }^{5}$ Cheng-Jeng Tai, ${ }^{2,3}$ \\ Chia-Wowi Wang, ${ }^{1,6}$ Yu-Jia Chang, ${ }^{7,8,9,10}$ Chen-Yen Choong, ${ }^{1,6}$ \\ Chi-Shian Lin, ${ }^{11}$ Chen-Jei Tai, ${ }^{1,4,12}$ and Chun-Chao Chang, \\ ${ }^{1}$ Department of Obstetrics and Gynecology, School of Medicine, College of Medicine, Taipei Medical University, Taipei 110, Taiwan \\ ${ }^{2}$ Division of Hematology and Oncology, Department of Internal Medicine, Taipei Medical University Hospital, Taipei 110, Taiwan \\ ${ }^{3}$ Department of Internal Medicine, School of Medicine, College of Medicine, Taipei Medical University, Taipei 110, Taiwan \\ ${ }^{4}$ Department of Chinese Medicine, Taipei Medical University Hospital, Taipei 110, Taiwan \\ ${ }^{5}$ Division of General Surgery, Department of Surgery, Chi-Mei Medical Center, Tainan 710, Taiwan \\ ${ }^{6}$ Department of Obstetrics and Gynecology, Taipei Medical University Hospital, Taipei 110, Taiwan \\ ${ }^{7}$ Cancer Research Center, Taipei Medical University and Hospital, Taipei 110, Taiwan \\ ${ }^{8}$ Department of Surgery, Taipei Medical University and Hospital, Taipei 110, Taiwan \\ ${ }^{9}$ Division of General Surgery, Department of Surgery, Taipei Medical University Hospital, Taipei Medical University, Taipei 110, Taiwan \\ ${ }^{10}$ Graduate Institute of Clinical Medicine, College of Medicine, Taipei Medical University, Taipei 110, Taiwan \\ ${ }^{11}$ Graduate Institute of Medical Sciences, College of Medicine, Taipei Medical University, Taipei 110, Taiwan \\ ${ }^{12}$ Traditional Herbal Medicine Research Center, Taipei Medical University Hospital, Taipei 110, Taiwan \\ ${ }^{13}$ Division of Gastroenterology and Hepatology, Department of Internal Medicine, Taipei Medical University Hospital, \\ Taipei 110, Taiwan
}

Correspondence should be addressed to Chen-Jei Tai; chenjtai@tmu.edu.tw and Chun-Chao Chang; chunchao@tmu.edu.tw

Received 26 September 2014; Revised 26 December 2014; Accepted 26 December 2014

Academic Editor: Mohammad Ahmad Al-Shatouri

Copyright (C) 2015 Chien-Kai Wang et al. This is an open access article distributed under the Creative Commons Attribution License, which permits unrestricted use, distribution, and reproduction in any medium, provided the original work is properly cited.

\begin{abstract}
Chemotherapy is the main approach for treating advanced and recurrent hepatocellular carcinoma (HCC), but the clinical performance of chemotherapy is limited by a relatively low response rate, drug resistance, and adverse effects that severely affect the quality of life of patients. The aqueous extract of Solanum nigrum (AE-SN) is a crucial ingredient in some traditional Chinese medicine (TCM) formulas for treating cancer patients and exhibits antitumor effects in human HCC cells. Therefore, this study examined the tumor-suppression efficiency of AE-SN integrated with a standard chemotherapeutic drug, namely, cisplatin or doxorubicin, in human HCC cells, namely, Hep3B and HepJ5. The results suggested that the integrated treatment with AE-SNpotentiated cisplatin and doxorubicin induced cytotoxicity through the cleavage of caspase-7 and accumulation of microtubuleassociated protein-1 light chain-3 A/1B II (LC-3 A/B II), which were associated with apoptotic and autophagic cell death, respectively, in both the Hep3B and HepJ5 cells. In conclusion, AE-SN can potentially be used in novel integrated chemotherapy with cisplatin or doxorubicin to treat HCC patients.
\end{abstract}

\section{Introduction}

Liver cancer is one of the most common malignant diseases worldwide, particularly in eastern Asia and sub-Saharan
Africa, and hepatocellular carcinoma (HCC) is the most prevalent type of liver cancer [1]. A major challenge in treating HCC is the poor prognosis for advanced and recurrent cases. 
Although chemotherapy is the main approach used to treat advanced and recurrent HCC cases, its clinical performance is largely limited by various factors such as a relatively low response rate, drug resistance, and various adverse effects that substantially impact the quality of life (QOL) of HCC patients [2]. The development of complementary and alternative medicines for improving the tumor-suppression efficiency of current chemotherapeutic drugs and managing the QOL of HCC patients has become an accepted optional approach worldwide $[3,4]$. Traditional Chinese medicine (TCM) has long been employed in treating various cancers through the use of numerous herb-based formulas; however, most of these formulas lack sufficient, basic clinical medical evidence verifying their antitumor efficacy.

TCM formulas are normally prepared using mixed extracts, with the composition and dose of the ingredients sometimes varying among individual cases. The varying composition and dosage cause difficulty in clarifying the antitumor efficacy of the formulas in clinical trials and experimental studies [2]. An alternative approach is examining the individual ingredients from specific TCM formulas that may contribute to the tumor-suppression efficacy. For instance, recent studies have suggested that certain crude extracts in TCM formulas, such as extracts of Semen Coicis, Scutellaria barbata, and Solanum nigrum, exhibited tumor-suppression efficacy in human HCC cells [5-7]. In recent studies, total flavonoids extracted from Scutellaria barbata inhibited cell proliferation and invasion of hepatocarcinoma via mediation of matrix metalloproteinases and metalloproteinases [8], and saikosaponin-D extracted from Bupleurum chinense DC also was reported to enhance radio sensitivity on hepatoma cells by adjusting cell cycle or hypoxic conditions $[9,10]$. These findings suggested that components and crude extracts of some TCM herbs may inhibit hepatocarcinoma cells via various mechanisms. The crude extracts of Solanum nigrum have demonstrated antitumor effects in various types of cancer, including human melanoma and colorectal, endometrial, cervical, and breast cancers [11-15]. Previous studies have indicated that the aqueous extract of Solanum nigrum leaves (AE-SN) mainly suppressed tumor cell growth by activating programmed cell death associated with caspase-3-dependent apoptosis [7] and LC-3 A/B-related autophagy [7, 11, 12, 14]. In addition, AE-SN is capable of enhancing the cytotoxicity induced by various chemotherapeutic drugs, including cisplatin, doxorubicin, and docetaxel, in human endometrial and colorectal cancer cells $[11,12]$, suggesting that AE-SN is a potential ingredient to develop for integrated chemotherapy with standard chemotherapeutic drugs. Because cisplatin and doxorubicin are the standard therapeutic drugs for treating HCC cases, knowing the antitumor effects of AE-SN in combination with either cisplatin or doxorubicin in human HCC cells would be beneficial.

To understand the potential of AE-SN for use in integrated chemotherapy with cisplatin or doxorubicin in human HCC cells, the main aim of the present study was to clarify whether AE-SN enhances the cytotoxicity induced by cisplatin and doxorubicin in human HCC cells. The results showed that a single treatment with AE-SN activated programmed cell death and provides insight into the efficacy of integrating AE-SN with chemotherapeutic drugs in treating HCC cells. The study results provide experimental evidence for supporting further application of AE-SN in HCC therapy.

\section{Materials and Methods}

2.1. Cell Lines and Regents. Two human HCC cell lines, namely, Hep3B and HepJ5, and one normal human pulmonary fibroblast, namely, WI-38, were purchased from the Bioresource Collection and Research Center (Hsinchu, Taiwan). Hep3B is an HCC cell line commonly used to examine antitumor components, and, by comparison, HepJ5 is a more malignant and resistant cell line that exhibits high expression of survivin [16]. This study used these two HCC cell lines to examine the antitumor effects of AE-SN and used WI-38 cells to examine the cytotoxicity of AE-SN in normal human cells. All of the cell lines were maintained in Dulbecco's modified Eagle's medium (Gibco, Grand Island, NY, USA) in addition to $100 \mathrm{U} / \mathrm{mL}$ of penicillin and $100 \mu \mathrm{g} / \mathrm{mL}$ of streptomycin (Invitrogen Life Technologies, Carlsbad, CA, USA) at $37^{\circ} \mathrm{C}$ in a $5 \% \mathrm{CO}_{2}$ humidified incubator. Cisplatin and doxorubicin were purchased from Sigma-Aldrich (St. Louis, MO, USA). The cell lysis buffer was prepared using a solution of $150 \mathrm{mM} \mathrm{NaCL}, 50 \mathrm{mM}$ Tris-HCL (pH 7.5), 1\% NP-40, 0.5\% deoxycholate, $0.1 \%$ SDS, $1 \mathrm{mM}$ PMSF, $10 \mu \mathrm{g} / \mathrm{mL}$ of leupeptin, and $100 \mu \mathrm{g} / \mathrm{mL}$ of aprotinin. The primary antibodies used to detect protein expression and activation were LC-3 A/B, caspase-3, and caspase-7 (Cell Signaling Technology, Danvers, MA, USA) as well as glyceraldehyde 3-phosphate dehydrogenase (GAPDH, AbFrontier, Seoul, South Korea). A secondary antibody, donkey anti-rabbit horseradish peroxidase conjugate, was purchased from Santa Cruz Biotechnology (Santa Cruz, CA, USA). HPLC-grade acetonitrile (ACN) and methanol were purchased from JT Baker (Phillipsburg, NJ, USA). The purified solamargine was purchased from Tauto Biotech (Shanghai, China).

AE-SN was prepared using the TCM processing method to simulate the AE-SN administered to patients in TCM clinical practice. In brief, $50 \mathrm{~g}$ of dried Solanum nigrum leaves was immersed in $750 \mathrm{~mL}$ of distilled water, gradually heated to $100^{\circ} \mathrm{C}$, and maintained at $100^{\circ} \mathrm{C}$ for $1 \mathrm{~h}$. The $\mathrm{AE}-\mathrm{SN}$ solution was further condensed to a final concentration of $1 \mathrm{~g}$ of raw material $/ \mathrm{mL}$ through heating at $100^{\circ} \mathrm{C}$. The final AE-SN stock solution $(1 \mathrm{~g} / \mathrm{mL})$ was filtered using a $0.22 \mu \mathrm{m}$ filter before the experiment.

2.2. Quantitative Analysis of Solamargine in AE-SN. Solamargine is considered as a crucial antitumor component that existed in Solanum nigrum and can be a marker component for AE-SN [17]. The concentration of solamargine was therefore determined in the AE-SN stock solution by using liquid chromatography-mass spectrometry (LC-MS/MS) analysis. Before analysis, $100 \mu \mathrm{L}$ AE-SN stock solution was mixed with $900 \mu \mathrm{L}$ mixture of water: methanol $(30: 70, \mathrm{v} / \mathrm{v})$. LCMS/MS analysis was performed in the mobile phase of HPLC-grade acetonitrile (ACN) and deionized water with a Syncronis C18 column (Thermo Scientific, MA, USA). The flow rate is $0.5 \mathrm{~mL} / \mathrm{min}$ with splitted $0.25 \mathrm{~mL} / \mathrm{min}$ to mass. The ionization mode of the mass spectrometry condition was 


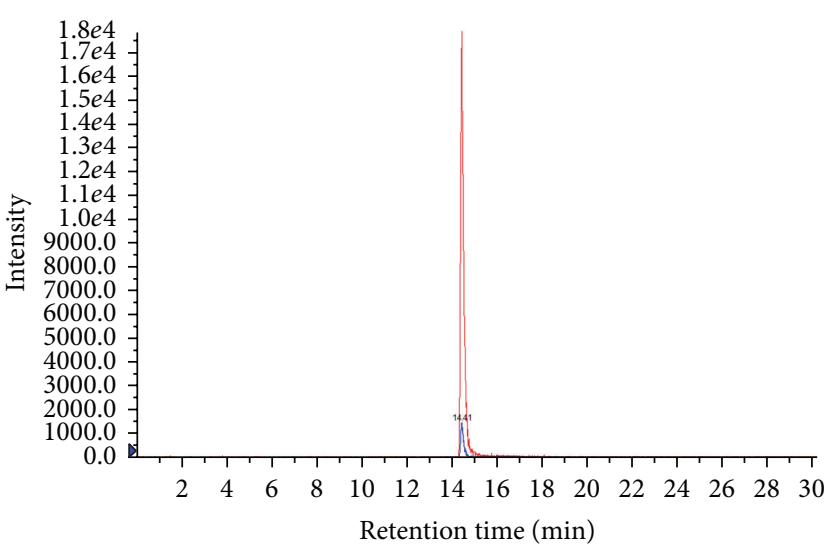

(a)

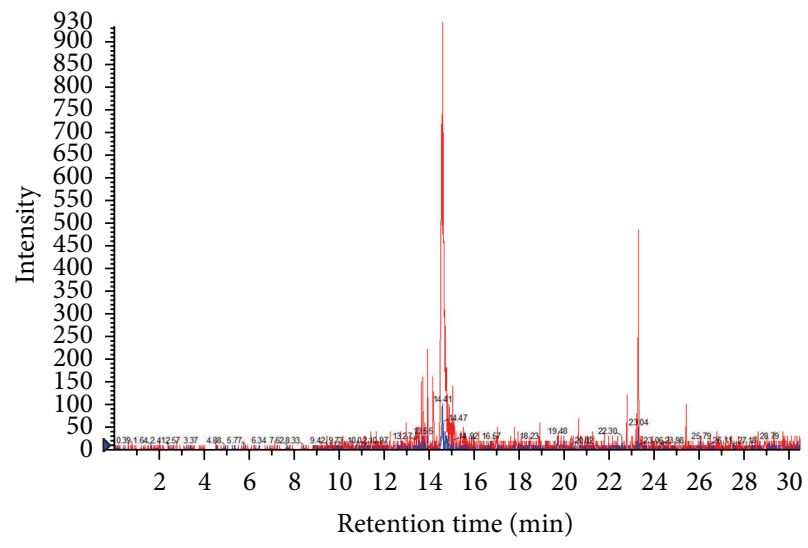

(b)

FIGURE 1: Extracted ion chromatography of solamargine. (a) The parent ion of purified solamargine was fragmented into two daughter ions (blue and red peaks). (b) Fragmented ions presented in AE-SN.

set as electrospray/positive ionization, and the mass scanning mode was multiple reaction monitor (MRM). The parent ion of purified solamargine is $867.5 \mathrm{~m} / \mathrm{z}$ and fragmented into two daughter ions: 722.5 and $396.4 \mathrm{~m} / z$, respectively (Figure $1(\mathrm{a})$ ). These daughter ions were used to determine the solamargine concentration in AE-SN (Figure 1(b)). The concentration of solamargine in AE-SN stock solution was $77 \mu \mathrm{g} / \mathrm{mL}$ by using MRM analysis.

\subsection{Cell Viability Assay and Morphological Observation.} Hep3B and HepJ5 cells were plated onto 96-well plates, with $5 \times 10^{3}$ cells per well, and cultured overnight before treatment. To evaluate the antitumor effects of AE-SN, the cells were treated with 0 to $2.0 \mathrm{mg} / \mathrm{mL}$ of AE-SN for $48 \mathrm{~h}$. To evaluate the antitumor effects of integrated treatment with the chemotherapeutic drugs and AE-SN, the cells were treated with 0 to $20 \mu \mathrm{M}$ cisplatin or 0 to $10 \mu \mathrm{M}$ and $0,0.5$, or $1.0 \mathrm{mg} / \mathrm{mL}$ of AE$\mathrm{SN}$ for $48 \mathrm{~h}$. In this study, cell viability was determined using a 3-(4,5-dimethylthiazol-2-yl)-2,5-diphenyltetrazolium bromide (MTT) assay (Table 1).

Two approaches, namely, microscopic observation and measurement of the cell size distribution, were performed to inspect the morphological changes in AE-SN-treated HCC cells. General morphological changes were observed using a Nikon Eclipse TS100 optical microscope (Nikon Instruments, Melville, NY, USA) and photographed at 100x magnification, whereas the distribution of cell diameter was measured using a Scepter cell counter (Merck Millipore Billerica, MA, USA), which divided surviving cells from cell fragments and debris in a borderline of $12 \mu \mathrm{m}$ [18].

2.4. Western Blotting Analysis. Hep3B and HepJ5 cells were planted into $6 \mathrm{~cm}$ dishes with $5 \times 10^{5}$ cells per dish and cultured overnight. The cells were treated with a control medium, namely, $5 \mu \mathrm{M}$ of cisplatin or $2 \mu \mathrm{M}$ of doxorubicin, and 0 or $1.0 \mathrm{mg} / \mathrm{mL}$ of AE-SN for $48 \mathrm{~h}$. After $48 \mathrm{~h}$ of incubation, the cells were collected using a cell lysis buffer. The protein concentrations of the cell lysates were determined using
TABLE 1: $\mathrm{IC}_{50}$ values in HCC cells treated with AE-SN alone or in combination with AE-SN and either chemotherapeutic drug. The $\mathrm{IC}_{50}$ values were analyzed using cell viability data determined after $48 \mathrm{~h}$ treatment with AE-SN (Figure 2) and with AE-SN integrated with either chemotherapeutic drug (Figure 3).

\begin{tabular}{lcccc}
\hline & \multicolumn{2}{c}{ Hep3B } & \multicolumn{2}{c}{ Hep5J } \\
\hline AE-SN $(\mathrm{mg} / \mathrm{mL})$ & \multicolumn{3}{c}{0.96} & \multicolumn{2}{c}{0.97} \\
\hline & 0 & 0.5 & 0 & 0.5 \\
\hline Cisplatin $(\mu \mathrm{M})$ & 6.75 & 2.74 & 8.71 & 2.84 \\
Doxorubicin $(\mu \mathrm{M})$ & 4.65 & 1.31 & 6.39 & 1.42 \\
\hline
\end{tabular}

a Bio-Rad protein assay kit (Bio-Rad Laboratories, Hercules, CA, USA) and equalized before western blotting analysis. The cell lysates were separated using $12.5 \%$ sodium dodecyl sulfate polyacrylamide gel electrophoresis and transferred into a polyvinylidene fluoride membrane (Pall Corp, Port Washington, NY, USA).

The expression and activation of the selected protein markers, namely, LC-3 A/B, caspase-3, and caspase-7, as well as the internal control, GAPDH, were then determined using corresponding primary and secondary antibodies. The immunoreactivity was detected using an electrochemiluminescence western blotting detection kit (Western Lightning Plus-ECL, PerkinElmer Inc., Waltham, MA, USA).

2.5. Statistical Analysis. Analyses of the half maximum inhibitory concentration $\left(\mathrm{IC}_{50}\right)$ were performed using CalcuSyn software (Biosoft, Cambridge, UK), whereas Student's $t$ test and one-way ANOVA were performed using SPSS software (SPSS Inc., Chicago, IL, USA).

\section{Results}

3.1. Cytotoxicity of AE-SN Alone and with the Chemotherapeutic Drugs in Hep3B and Hep5J Cells. In this study, 


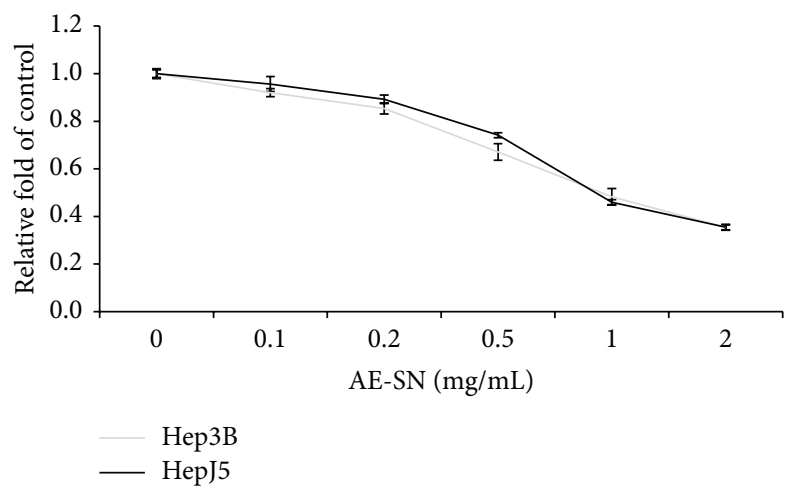

(a)
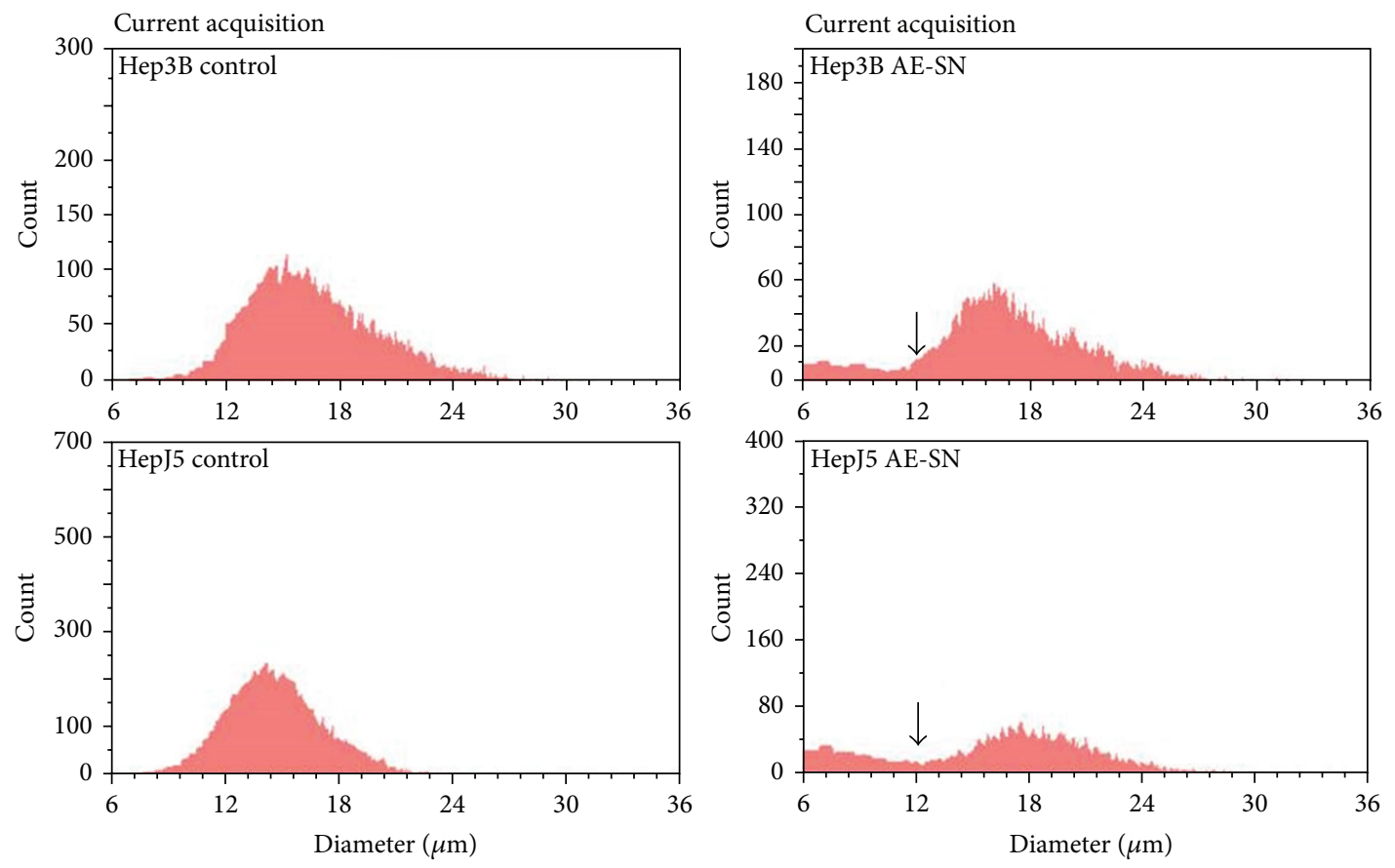

(b)

Figure 2: AE-SN treatment inhibited Hep3B and HepJ5 cell growth. (a) Hep3B and HepJ5 cells were treated with 0.1 to $2 \mathrm{mg} / \mathrm{mL}$ of AE-SN for $48 \mathrm{~h}$, and the cell viability was determined using an MTT assay. The data are presented as the mean \pm standard deviation. (b) Hep3B and HepJ 5 cells were treated with $1.0 \mathrm{mg} / \mathrm{mL}$ of AE-SN for $48 \mathrm{~h}$, and the cell size distribution was determined according to the cell diameter by using a Scepter cell counter. Arrows indicate a cell diameter of $12 \mu \mathrm{m}$.

$48 \mathrm{~h}$ treatment with 0 to $2.0 \mathrm{mg} / \mathrm{mL}$ of $\mathrm{AE}-\mathrm{SN}$ gradually inhibited the growth of Hep3B and HepJ5 cells in a concentration-dependent manner (one-way ANOVA, $P<0.01$, Figure 2(a)). The $\mathrm{IC}_{50}$ values of $\mathrm{AE}-\mathrm{SN}$ for the Hep3B and HepJ5 cells were 0.96 and $0.97 \mathrm{mg} / \mathrm{mL}$, respectively. AE-SN treatment also resulted in the production of cell fragments and debris, which were less than $12 \mu \mathrm{m}$ in diameter in comparison with cells treated with the control medium in both Hep3B and HepJ5 cells (Figure 2(b)). Collectively, these results suggested that $\mathrm{AE}-\mathrm{SN}$ inhibited cell growth and demonstrated cytotoxicity in Hep3B and HepJ5 cells.

To evaluate cytotoxicity on the integrated treatment with chemotherapeutic drugs and AE-SN, cisplatin (1 to $20 \mu \mathrm{M})$ or doxorubicin (1 to $10 \mu \mathrm{M})$ was used to treat the Hep3B and
HepJ5 cells, respectively, with $0,0.5$, or $1.0 \mathrm{mg} / \mathrm{mL}$ of AE-SN for $48 \mathrm{~h}$ (Figure 3 ). The $\mathrm{IC}_{50}$ values of the cisplatin integrated with $0.5 \mathrm{mg} / \mathrm{mL}$ of AE-SN in the Hep3B and HepJ5 cells were reduced to $2.74 \mu \mathrm{M}$ and $2.84 \mu \mathrm{M}$, respectively, whereas those of doxorubicin were reduced to $1.31 \mu \mathrm{M}$ and $1.42 \mu \mathrm{M}$. In other words, the $\mathrm{IC}_{50}$ values for cisplatin and doxorubicin integrated with $0.5 \mathrm{mg} / \mathrm{mL}$ of AE-SN were reduced to $40 \%$ and $30 \%$ of the values of the drugs used alone in the Hep3B and $\mathrm{J} 5$ cells, respectively. Human pulmonary fibroblast cells, WI-38, were also treated with $0,0.5$, or $1.0 \mathrm{mg} / \mathrm{mL}$ of AE$\mathrm{SN}$ and cisplatin or doxorubicin to identify the cytotoxicity of the combined treatment (Figures 3(c) and 3(f)). AE-SN was not likely to enhance cisplatin- or doxorubicin-induced cytotoxicity in the WI-38 cells in comparison with that in the 

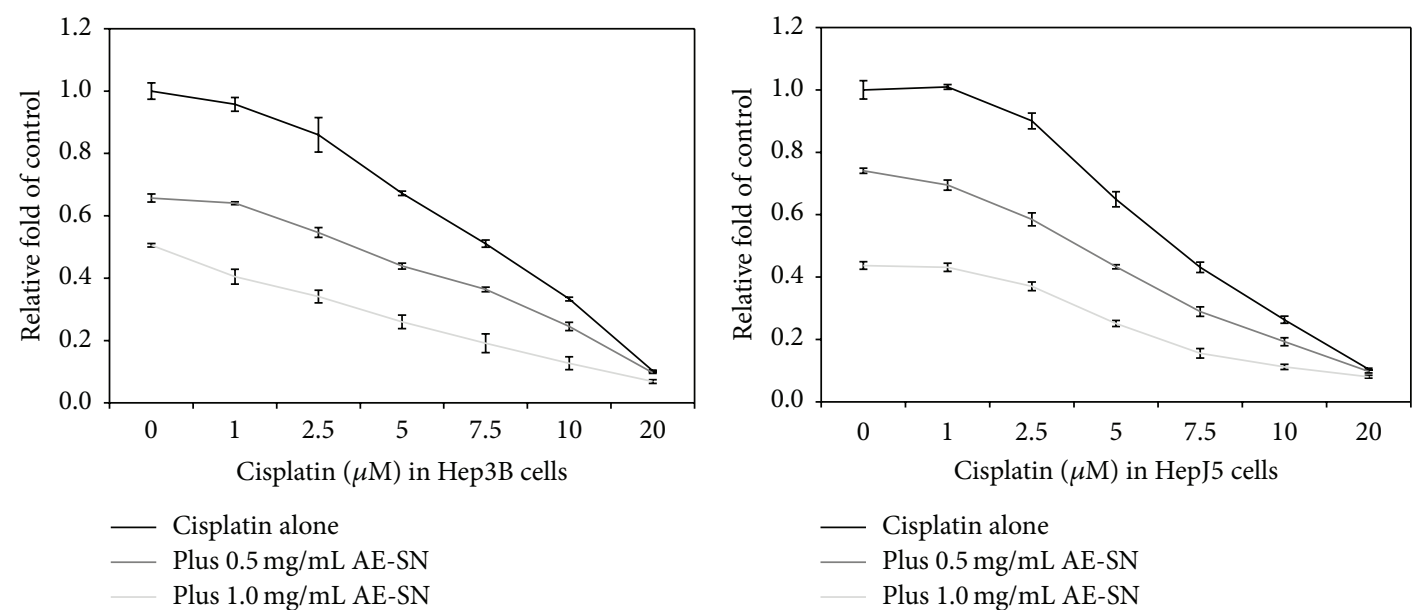

(b)
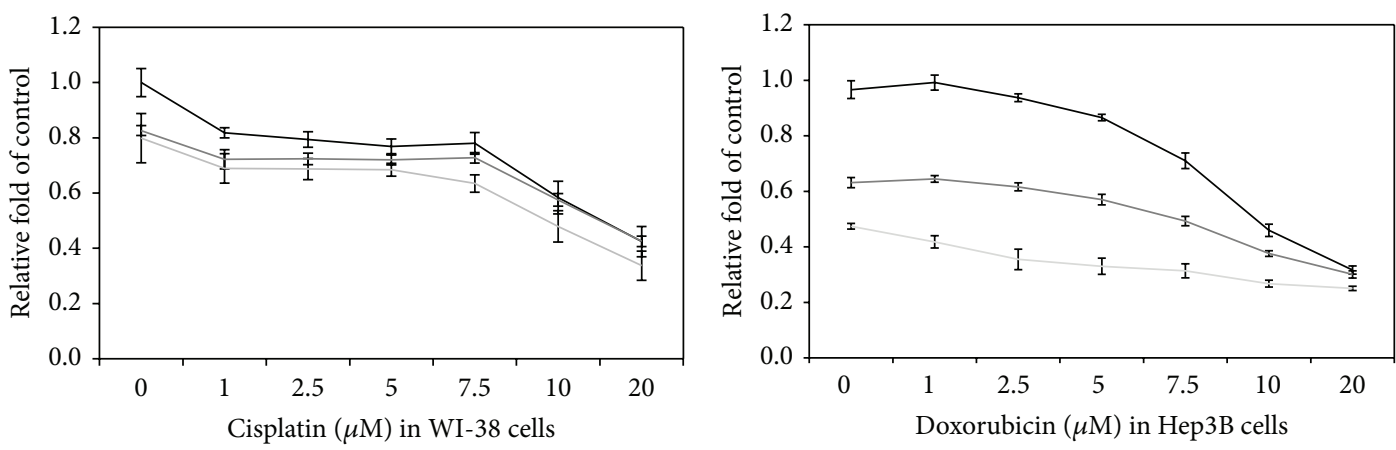

Plus $0.5 \mathrm{mg} / \mathrm{mL}$ AE-SN
Plus $1.0 \mathrm{mg} / \mathrm{mL}$ AE-SN

(c)

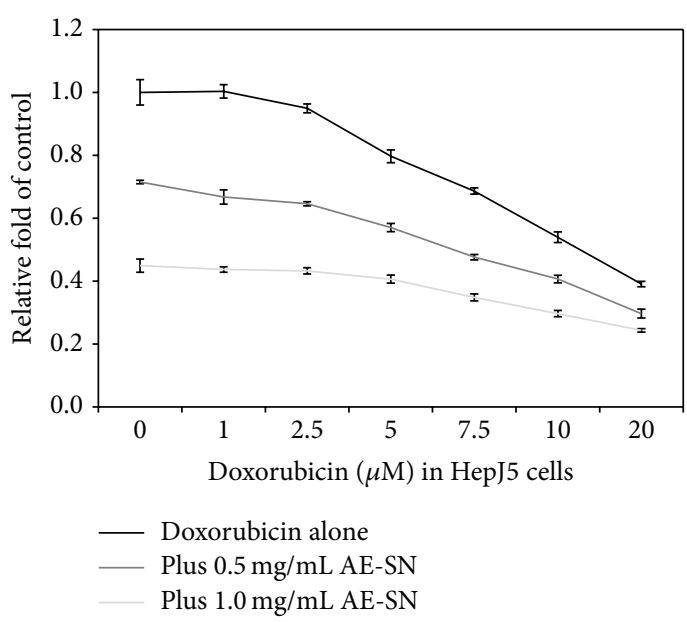

(e)
_ Doxorubicin alone

- Plus $0.5 \mathrm{mg} / \mathrm{mL}$ AE-SN Plus $1.0 \mathrm{mg} / \mathrm{mL}$ AE-SN

(d)

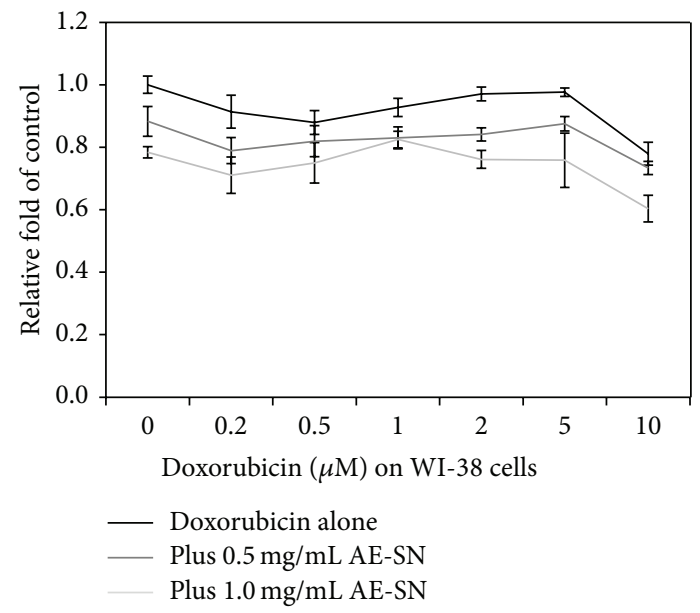

(f)

FIGURE 3: AE-SN-potentiated cisplatin and doxorubicin induced cytotoxicity in Hep3B and HepJ5 cells but had no effect on normal human pulmonary fibroblasts (WI-38 cells). (a-c) Cells were treated with 0 to $20 \mu \mathrm{M}$ cisplatin and $0,0.5$, or $1.0 \mathrm{mg} / \mathrm{mL}$ of AE-SN for $48 \mathrm{~h}$. (d-f) Cells were treated with 0 to $10 \mu \mathrm{M}$ doxorubicin and $0,0.5$, or $1.0 \mathrm{mg} / \mathrm{mL}$ of AE-SN for $48 \mathrm{~h}$. The cell viability was determined using an MTT assay; the data are presented as the mean \pm standard deviation. 


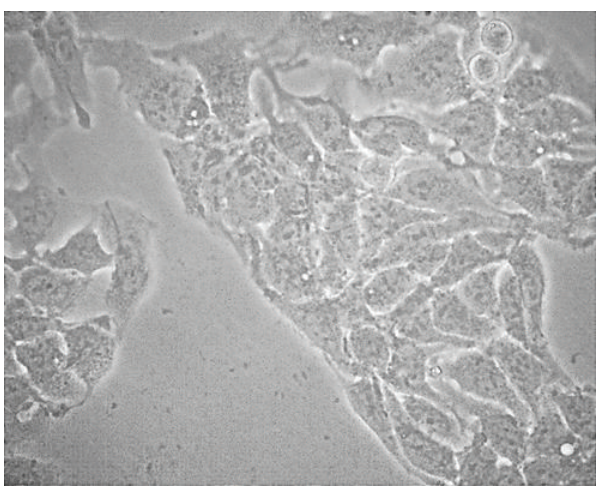

(a)

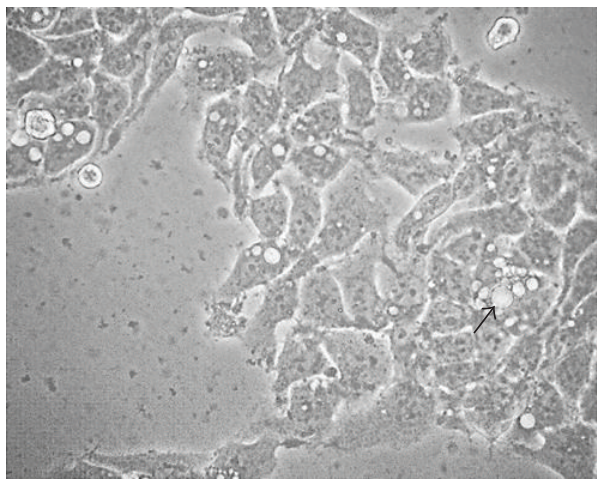

(c)

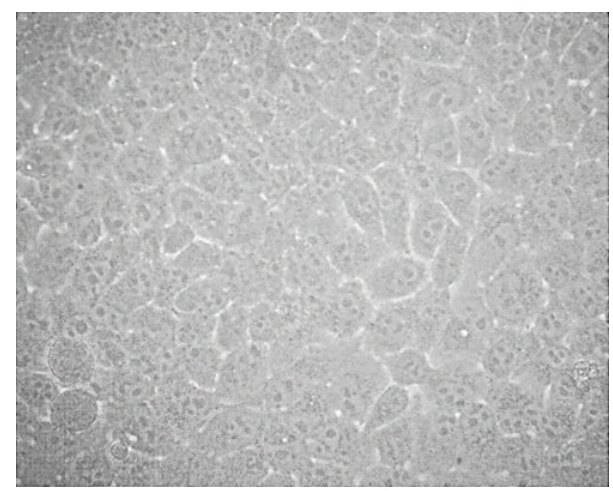

(b)

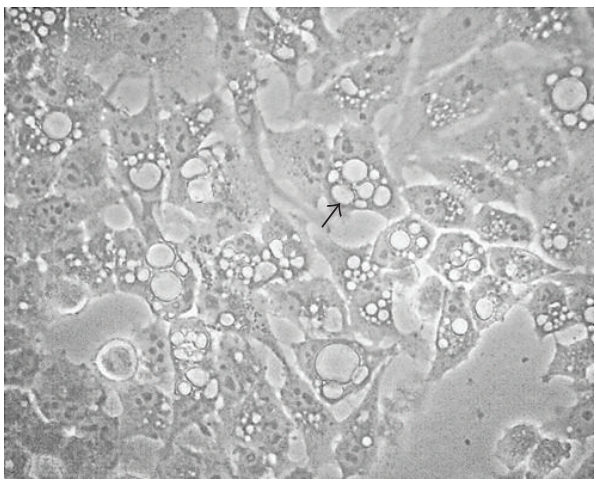

(d)

Figure 4: AE-SN-activated programmed cell death in Hep3B and HepJ5 cells. (a) Hep3B and (b) HepJ5 cells were treated with a control medium for $48 \mathrm{~h}$. (c) Hep3B and (d) HepJ5 cells were treated with $1.0 \mathrm{mg} / \mathrm{mL}$ of AE-SN for $48 \mathrm{~h}$. Arrows indicate the morphological changes present in the AE-SN-treated cells (100x magnification).

Hep3B and HepJ5 cells. Together, these results suggested that the AE-SN treatment potentiated cisplatin- and doxorubicininduced cytotoxicity in both Hep3B and HepJ5 cells but not in WI-38 normal human cells.

\subsection{Activation of Programmed-Cell-Death-Related Protein} in AE-SN and Chemotherapeutic-Drug-Treated Cancer Cells. Hep3B and HepJ5 cells treated with AE-SN demonstrated a clear morphological change similar to the formation of phagolysosome-like vacuoles (Figures 4(a) to 4(d)). This morphological change was also observed in AE-SN-treated human endometrial and colorectal carcinoma cells [11] and related to the activation of autophagy.

To further understand whether AE-SN increases the activation of cell death protein when combined with either cisplatin or doxorubicin in the two HCC cell lines, the activation of LC-3 A/B and caspase-7 was observed in HCC cells treated with $1 \mathrm{mg} / \mathrm{mL}$ of AE-SN and $5 \mu \mathrm{M}$ cisplatin or $2 \mu \mathrm{M}$ doxorubicin for $48 \mathrm{~h}$. As shown in Figure 5, the results suggested that, in both Hep3B and HepJ5 cells, AE-SN induced LC-3 A/B II accumulation in all of the treatment groups. The cleavage of caspase-7 was also enhanced by AE-SN cotreatment in cisplatin-treated Hep3B and HepJ5 cells and in doxorubicintreated HepJ5 cells, but not in doxorubicin-treated Hep3B cells. Doxorubicin cotreatment seemed to eliminate the AE$\mathrm{SN}$-induced caspase-7 cleavage in the Hep3B cells.

\section{Discussion}

In this study, the impact of AE-SN on tumor-suppression efficiency was evaluated by employing the preparation of AE$\mathrm{SN}$ that is used in TCM clinical practice. The $\mathrm{IC}_{50}$ values of AE-SN for Hep3B and HepJ5 cells indicated a moderate antitumor effect through direct exposure. Our previous studies showed that administering AE-SN at approximately 0.5 to $1 \mathrm{mg} / \mathrm{mL}$ for $48 \mathrm{~h}$ yielded similar $\mathrm{IC}_{50}$ values in human endometrial and colorectal cancer cells $[11,12]$. In comparison with the tumor-suppression efficiency observed in previous studies using AE-SN extracts prepared from dried powder through water extraction $[7,13,14,19,20]$, the tumorsuppression efficiency of AE-SN in this study was similar. The results of these experimental studies collectively suggest that cancer treatment may be a reasonable application for the preparation of AE-SN used in TCM practice. In addition, integrated treatment of AE-SN with cisplatin and doxorubicin may substantially reduce the required dose of cisplatin and doxorubicin required to achieve the same tumorsuppression efficiency, thereby improving the QOL of HCC patients during chemotherapy.

AE-SN was suggested to activate both autophagic and apoptotic cell death in many human cancer cell lines by inducing the cleavage of caspase- 3 and accumulation of LC3 A/B II [7]. By contrast, this study indicated that AE-SN 


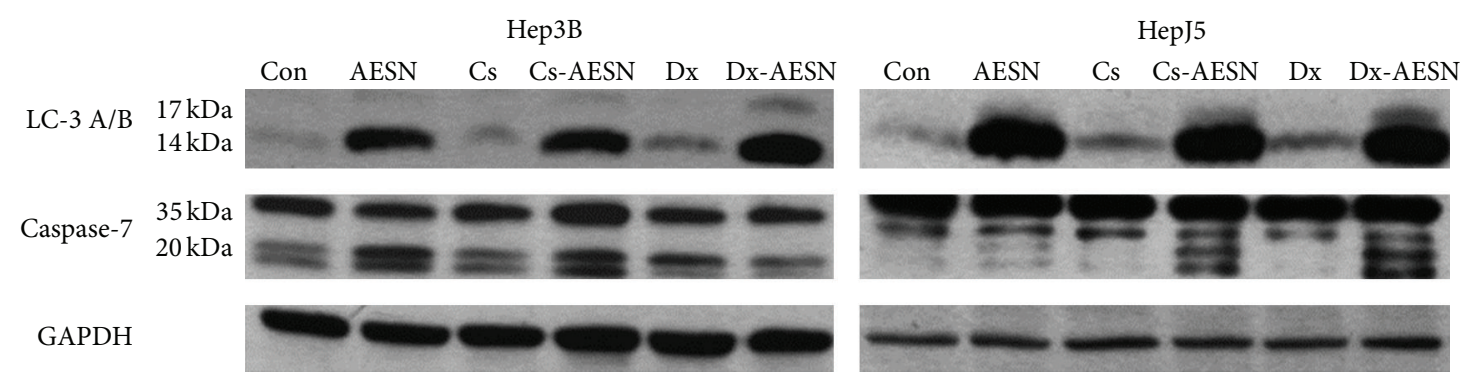

(a)
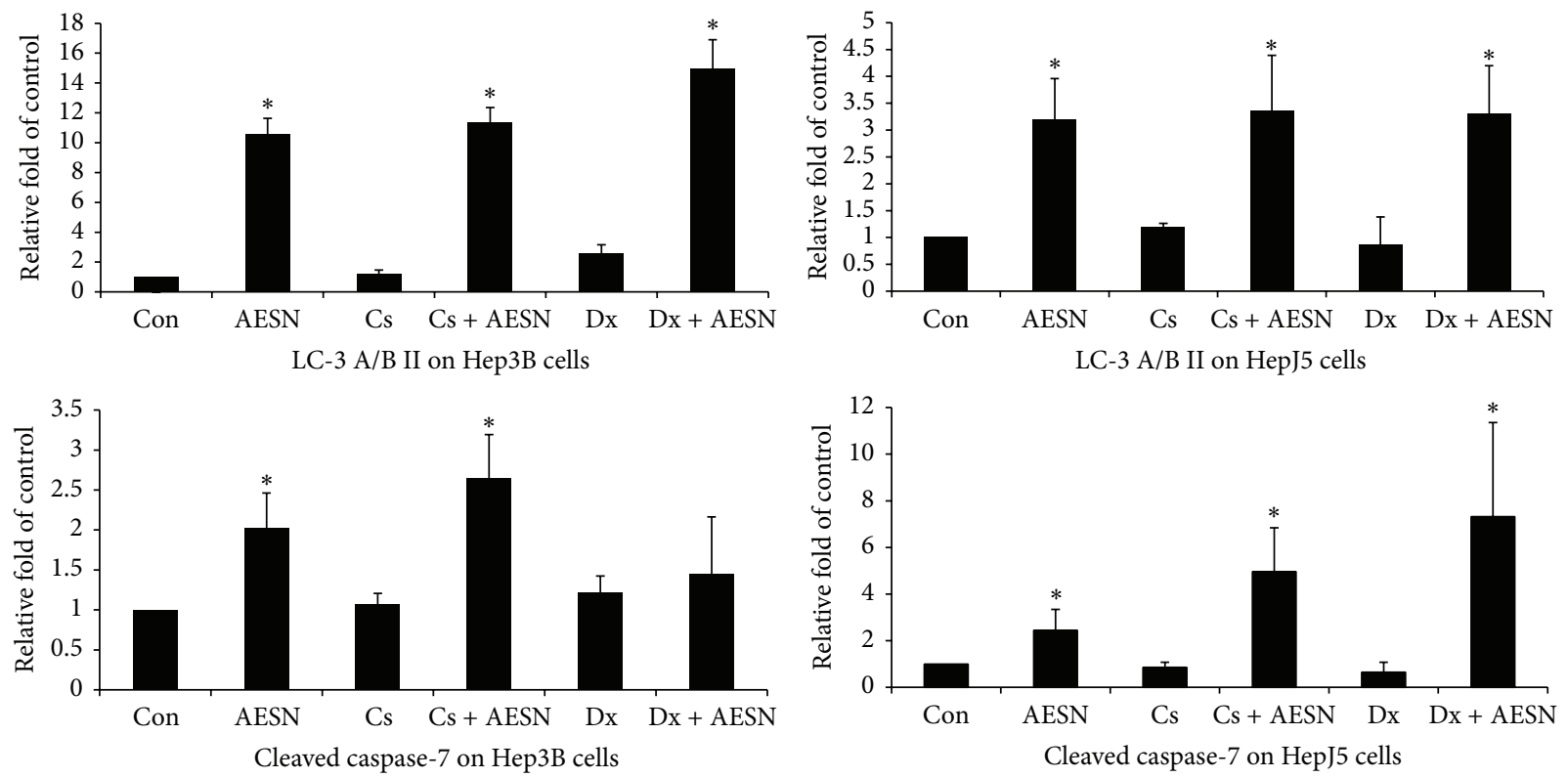

(b)

FIGURE 5: Activation of selected protein markers in HCC cells treated with AE-SN and either cisplatin or doxorubicin. (a) Cells were treated with a control medium, namely, $5 \mu \mathrm{M}$ cisplatin or $2 \mu \mathrm{M}$ doxorubicin with 0 or $1.0 \mathrm{mg} / \mathrm{mL}$ of AE-SN for $48 \mathrm{~h}$. The activation of LC-3 A/B and caspase-7 was determined using western blotting analysis. GAPDH served as an internal control. (b) Semiquantitation of the LC-3 A/B II and cleaved caspase- 7 in the Hep3B and HepJ5 cells. The data are presented as the mean \pm standard deviation. * indicates statistical significance in comparison with the previous group, namely, Con versus AESN (vehicle control versus AE-SN), Cs versus Cs + AESN, and Dx versus Dx + AESN (two-tailed Student's $t$-test, $P<0.05$ ).

treatment failed to induce the cleavage of caspase-3 in both Hep3B and HepJ5 cells (data not shown). This result coincided with observations of human colorectal and endometrial cancer cells $[11,12]$ that suggested that resistance to AE-SNinduced caspase-3 cleavage may vary among cancer cell types. Cleaved caspase-7 is another biomarker for apoptotic cell death and was found to be induced in Hep3B and HepJ5 cancer cells after a single treatment with AE-SN. The cleavage of caspase-7 can be induced through mitochondria-mediated and extracellular signal-induced apoptosis pathways [21]. AE-SN-induced caspase-7 cleavage may occur through an alternative signal pathway, independent of caspase-3 cleavage, and activates apoptosis in caspase-3 resistant cancer cells. In addition, AE-SN induces LC-3 A/B II accumulation, which is associated with autophagy activation in Hep3B and HepJ5 cells. These results have been a common feature among AE-SN studies $[7,11,12,14]$. In integrated treatment, AE-SN still induced both LC-3 A/B II accumulation and caspase-7 cleavage in both cisplatin- and doxorubicin-treated cells, suggesting that AE-SN may enhance cisplatin- and doxorubicin-induced cytotoxicity through the activation of autophagy and caspase-7-related apoptosis.

In comparison with Hep3B cells, HepJ5 cells are more malignant and resistant and exhibit a higher expression of survivin [16]. In the present study, the $\mathrm{IC}_{50}$ values for cisplatin and doxorubicin in HepJ5 cells were higher than those in Hep3B cells (8.71 versus 6.75 and 6.39 versus $4.65 \mu \mathrm{M}$, resp.). This result confirmed that HepJ5 cells are more resistant to cisplatin- and doxorubicin-induced cytotoxicity than Hep3B cells were. By contrast, the $\mathrm{IC}_{50}$ values for $\mathrm{AE}-\mathrm{SN}$ were similar in HepJ5 and Hep3B cells ( 0.97 versus $0.96 \mathrm{mg} / \mathrm{mL}$ ), and combined treatment with cisplatin or doxorubicin and $0.5 \mathrm{mg} / \mathrm{mL}$ of $\mathrm{AE}-\mathrm{SN}$ further reduced the $\mathrm{IC}_{50}$ values to the same levels (2.84 versus $2.74 \mu \mathrm{M}$ in the cisplatin combination and 1.31 versus $1.42 \mu \mathrm{M}$ in the doxorubicin combination). These results collectively suggested that HepJ5 cells were 


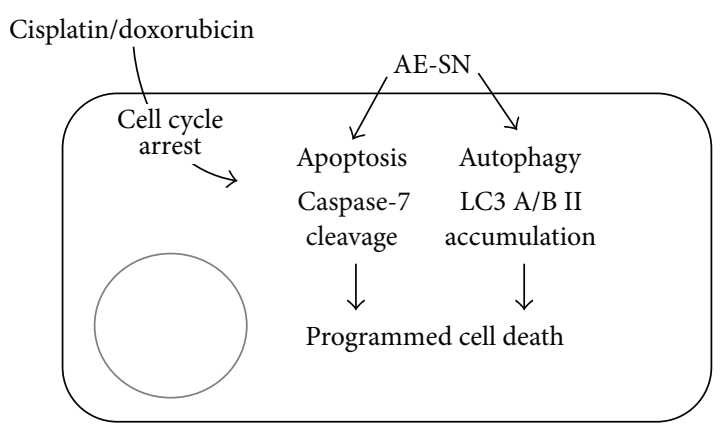

Hepatocellular carcinoma cell

FIGURE 6: Illustration of the integrated-cell-death mechanism activated by AE-SN and either cisplatin or doxorubicin in HCC cells.

unable to resist AE-SN-induced cytotoxicity and tended to be more vulnerable to cisplatin- and doxorubicin-induced cytotoxicity in combined treatment with AE-SN. The molecular mechanisms involved in this AE-SN-mediated HCC cytotoxicity require further investigation.

Some steroidal alkaloid glycosides and glycoproteins are considered the major active substances responsible for $\mathrm{AE}$ $\mathrm{SN}$-induced programmed cell death. Solamargine is one of the major compounds present in $\mathrm{AE}-\mathrm{SN}$ that can activate caspase-3-related apoptosis and LC-3-A/B-II-related autophagy in human leukemia cells [22], and a $150 \mathrm{kDa}$ glycoprotein isolated from $\mathrm{AE}-\mathrm{SN}$ was reported to be another effective antitumor compound for activating caspase-3-related apoptosis in human colorectal, cervical, and HCC cells [23-25]. However, these isolated compounds do not seem to completely explain the tumor-suppression mechanism of AE-SN because the specific compound that activates the cleavage of caspase-7 remains unknown. Further investigation is therefore required to identify the exact composition of the AE-SN substance that contributes to the antitumor effects.

This study clarified the antitumor effects of AE-SN and the potential of AE-SN for enhancing cytotoxicity induced by cisplatin and doxorubicin in human HCC cells in vitro. In consideration of the absorption rate of $\mathrm{AE}-\mathrm{SN}$ through the gastrointestinal tracts and its metabolism in vivo, the real tumor-suppression efficiency of AE-SN with chemotherapeutic drugs as well as the optimal dosage, preparation, and administrative approach of AE-SN remains to be evaluated using an animal cancer model. In addition, any unexpected adverse effects of integrated treatment with AE-SN and chemotherapeutic drugs in vivo should be carefully examined before clinical trials are conducted.

\section{Conclusion}

Clear experimental evidence obtained in this study indicates that the AE-SN cotreatment potentiated cisplatinand doxorubicin-induced cytotoxicity in human HCC cells. This AE-SN-potentiated cytotoxicity may occur through the accumulation of LC-3 A/B II and cleavage of caspase-7 to activate apoptosis and autophagic cell death in human HCC cells (Figure 6). Collectively, our results suggest that AE-SN can be used in novel integrated chemotherapy with cisplatin and doxorubicin to improve tumor-suppression efficiency in HCC treatment.

\section{Conflict of Interests}

The authors declare that there is no conflict of interests.

\section{Authors' Contribution}

Chien-Kai Wang and Yi-Feng Lin contributed equally to this study.

\section{Acknowledgments}

This study was supported in part by Taipei Medical University and Taipei Medical University Hospital (102-TMU-TMUH19).

\section{References}

[1] H. B. El-Serag, "Hepatocellular carcinoma," New England Journal of Medicine, vol. 365, no. 12, pp. 1118-1127, 2011.

[2] Z. Wang, J. Li, Y. Ji, P. An, S. Zhang, and Z. Li, “Traditional herbal medicine: a review of potential of inhibitory hepatocellular carcinoma in basic research and clinical trial," EvidenceBased Complementary and Alternative Medicine, vol. 2013, Article ID 268963, 7 pages, 2013.

[3] E. Ernst and B. R. Cassileth, "The prevalence of complementary/ alternative medicine in cancer: a systematic review," Cancer, vol. 83, no. 4, pp. 777-782, 1998.

[4] G. Yang, X. Li, L. Wang et al., "Traditional chinese medicine in cancer care: a review of case series published in the chinese literature," Evidence-Based Complementary and Alternative Medicine, vol. 2012, Article ID 751046, 8 pages, 2012.

[5] Z. J. Dai, X. J. Wang, Z. F. Li et al., "Scutellaria barbate extract induces apoptosis of hepatoma $\mathrm{H} 22$ cells via the mitochondrial pathway involving caspase-3," World Journal of Gastroenterology, vol. 14, no. 48, pp. 7321-7328, 2008.

[6] Y. Lu, L.-Q. Wu, Q. Dong, and C.-S. Li, "Experimental study on the effect of Kang-Lai-Te induced apoptosis of human hepatoma carcinoma cell HepG2," Hepatobiliary \& Pancreatic Diseases International, vol. 8, no. 3, pp. 267-272, 2009.

[7] H. M. Lin, H. C. Tseng, C. J. Wang et al., "Induction of autophagy and apoptosis by the extract of Solanum nigrum Linn in HepG2 cells," Journal of Agricultural and Food Chemistry, vol. 55, no. 9, pp. 3620-3628, 2007.

[8] Z.-J. Dai, B.-F. Wang, W.-F. Lu et al., "Total flavonoids of Scutellaria barbata inhibit invasion of hepatocarcinoma via MMP/TIMP in vitro," Molecules, vol. 18, no. 1, pp. 934-950, 2013.

[9] B.-F. Wang, X.-J. Wang, H.-F. Kang et al., "Saikosaponin-D enhances radiosensitivity of hepatoma cells under hypoxic conditions by inhibiting hypoxia-inducible factor- $1 \alpha$," Cellular Physiology and Biochemistry, vol. 33, no. 1, pp. 37-51, 2014.

[10] B.-F. Wang, Z.-J. Dai, X.-J. Wang et al., "Saikosaponin-d increases the radiosensitivity of smmc-7721 hepatocellular carcinoma cells by adjusting the $\mathrm{g} 0 / \mathrm{g} 1$ and $\mathrm{g} 2 / \mathrm{m}$ checkpoints of the cell cycle," BMC Complementary and Alternative Medicine, vol. 13, article 263, 2013. 
[11] C. J. Tai, C. K. Wang, Y. F. Lin et al., "Aqueous extract of solanum nigrum leaves induces autophagy and enhances cytotoxicity of cisplatin, doxorubicin, docetaxel, and 5-fluorouracil in human colorectal carcinoma cells," Evidence-Based Complementary and Alternative Medicine, vol. 2013, Article ID 514719, 12 pages, 2013.

[12] C.-J. Tai, C.-K. Wang, Y.-J. Chang, and C.-S. Lin, "Aqueous extract of Solanum nigrum leaf activates autophagic cell death and enhances docetaxel-induced cytotoxicity in human endometrial carcinoma cells," Evidence-based Complementary and Alternative Medicine, vol. 2012, Article ID 859185, 10 pages, 2012.

[13] H.-C. Wang, D.-H. Wu, Y.-C. Chang, Y.-J. Li, and C.-J. Wang, "Solanum nigrum Linn. Water extract inhibits metastasis in mouse melanoma cells in vitro and in vivo," Journal of agricultural and food chemistry, vol. 58, no. 22, pp. 11913-11923, 2010.

[14] H. C. Huang, K. Y. Syu, and J. K. Lin, "Chemical composition of Solanum nigrum linn extract and induction of autophagy by leaf water extract and its major flavonoids in AU565 breast cancer cells," Journal of Agricultural and Food Chemistry, vol. 58, no. 15, pp. 8699-8708, 2010.

[15] J. Li, Q. Li, T. Feng, and K. Li, "Aqueous extract of Solanum nigrum inhibit growth of cervical carcinoma (U14) via modulating immune response of tumor bearing mice and inducing apoptosis of tumor cells," Fitoterapia, vol. 79, no. 7-8, pp. 548$556,2008$.

[16] C.-S. Hung, H.-H. Liu, M.-T. Huang et al., "Knockdown survivin expression reduces the efficacy of curcumin treatment in hepatocellular carcinoma cells," Annals of Surgical Oncology, vol. 19, no. 11, pp. 3547-3555, 2012.

[17] K. Hu, H. Kobayashi, A. Dong, Y. Jing, S. Iwasaki, and X. Yao, "Antineoplastic agents III: steroidal glycosides from solanum nigrum," Planta Medica, vol. 65, no. 1, pp. 35-38, 1999.

[18] M. Tahara, T. Inoue, Y. Miyakura et al., "Cell diameter measurements obtained with a handheld cell counter could be used as a surrogate marker of $\mathrm{G} 2 / \mathrm{M}$ arrest and apoptosis in colon cancer cell lines exposed to SN-38," Biochemical and Biophysical Research Communications, vol. 434, no. 4, pp. 753-759, 2013.

[19] A. Nawab, V. S. Thakur, M. Yunus, A. A. Mahdi, and S. Gupta, "Selective cell cycle arrest and induction of apoptosis in human prostate cancer cells by a polyphenol-rich extract of Solanum nigrum," International Journal of Molecular Medicine, vol. 29, no. 2, pp. 277-284, 2012.

[20] H. C. Wang, P. J. Chung, C. H. Wu, K. P. Lan, M. Y. Yang, and C. J. Wang, "Solanum nigrum L. polyphenolic extract inhibits hepatocarcinoma cell growth by inducing G2/M phase arrest and apoptosis," Journal of the Science of Food and Agriculture, vol. 91, no. 1, pp. 178-185, 2011.

[21] G. S. Salvesen and S. J. Riedl, "Caspase mechanisms," Advances in Experimental Medicine and Biology, vol. 615, pp. 13-23, 2008.

[22] L. Sun, Y. Zhao, X. Li, H. Yuan, A. Cheng, and H. Lou, "A lysosomal-mitochondrial death pathway is induced by solamargine in human K562 leukemia cells," Toxicology in Vitro, vol. 24, no. 6, pp. 1504-1511, 2010.

[23] S.-J. Lee, J.-H. Ko, and K.-T. Lim, "Glycine- and proline-rich glycoprotein isolated from Solanum nigrum Linne activates caspase-3 through cytochrome c in HT-29 cells," Oncology Reports, vol. 14, no. 3, pp. 789-796, 2005.

[24] P.-S. Oh and K.-T. Lim, "HeLa cells treated with phytoglycoprotein $(150 \mathrm{kDa})$ were killed by activation of caspase 3 via inhibitory activities of NF- $\kappa \mathrm{B}$ and AP-1," Journal of Biomedical Science, vol. 14, no. 2, pp. 223-232, 2007.
[25] S.-J. Lee and K.-T. Lim, "Cell death signal by glycine- and proline-rich plant glycoprotein is transferred from cytochrome c and nuclear factor kappa B to caspase 3 in Hep3B cells," Journal of Nutritional Biochemistry, vol. 19, no. 3, pp. 166-174, 2008. 


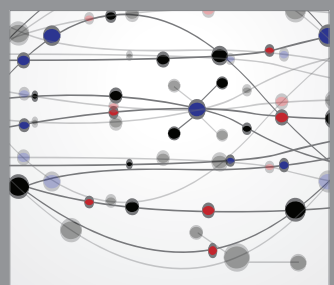

The Scientific World Journal
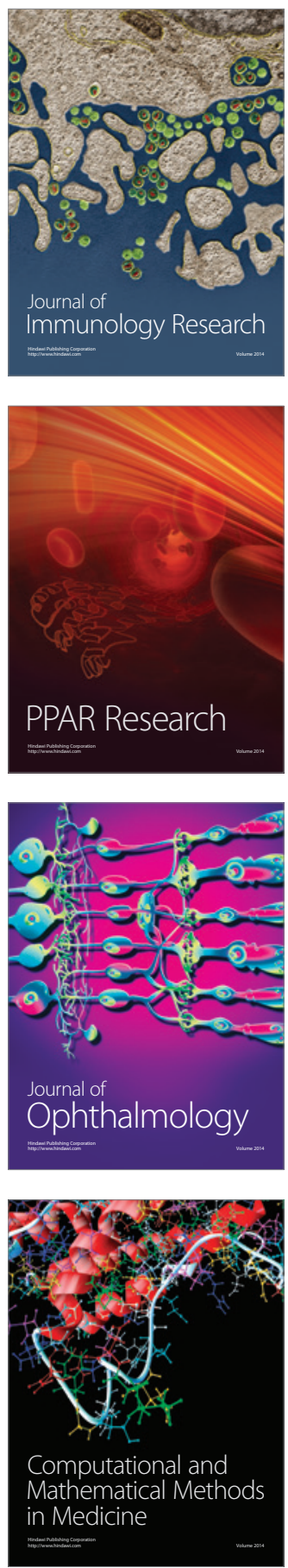

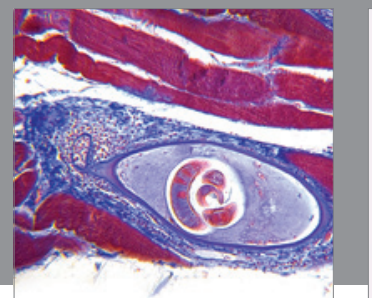

Gastroenterology

Research and Practice
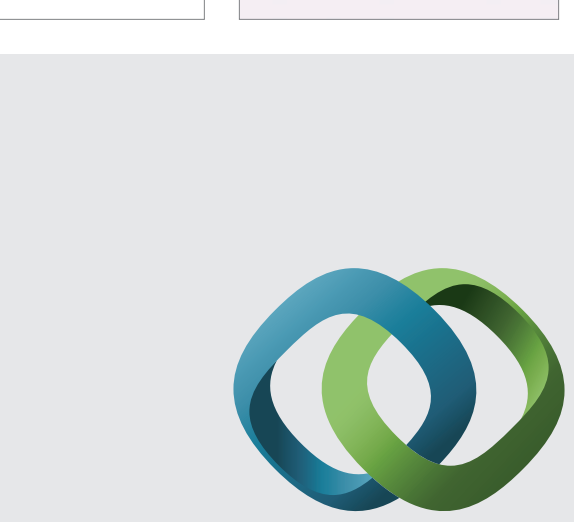

\section{Hindawi}

Submit your manuscripts at

http://www.hindawi.com
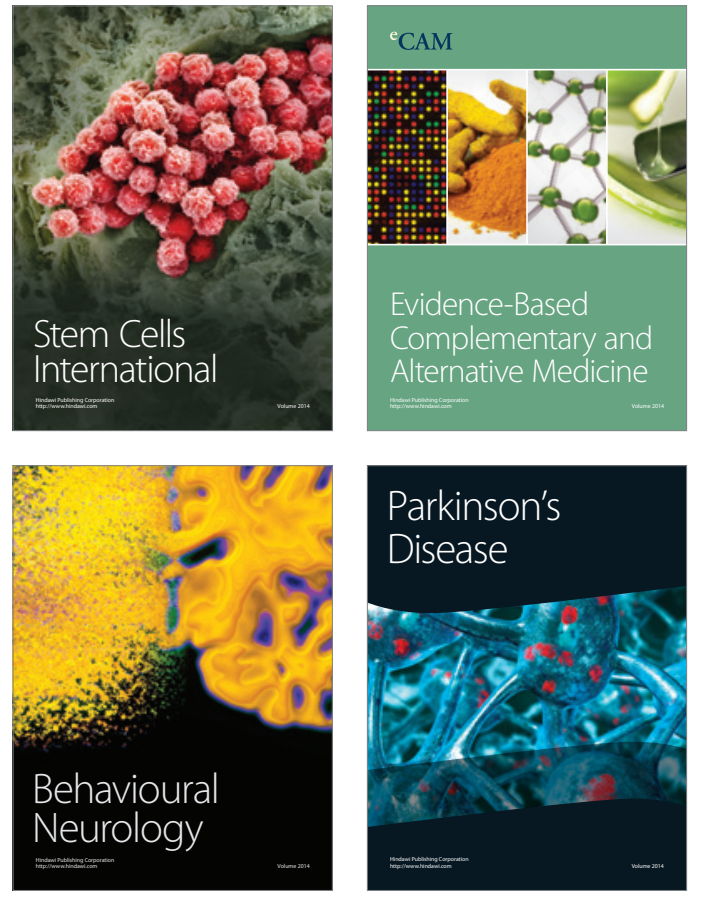
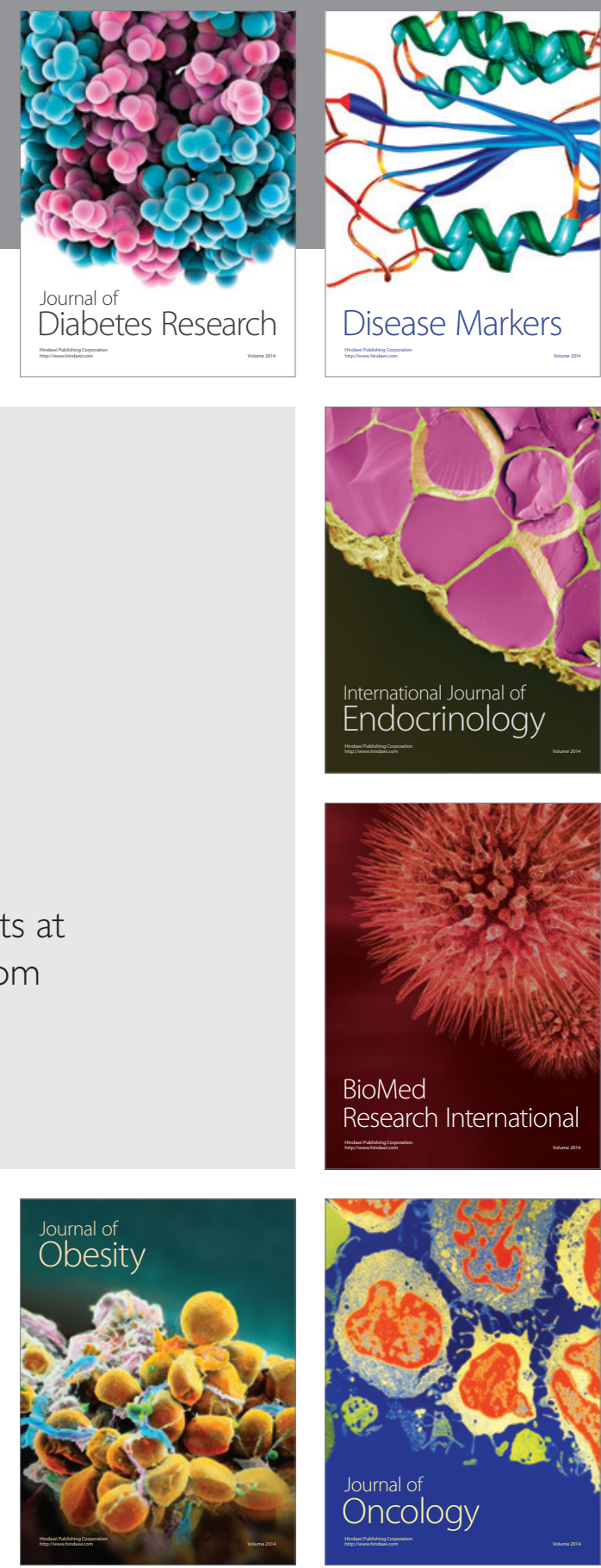

Disease Markers
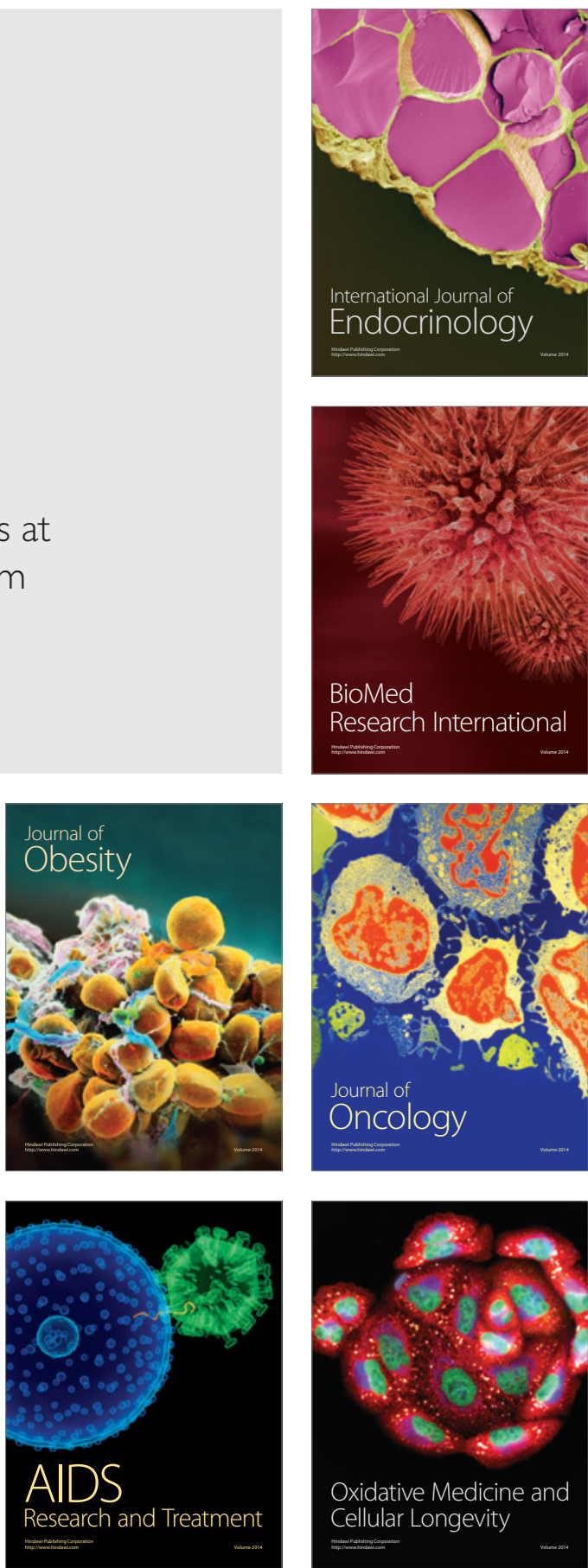